\title{
EDITORIAL
}

\section{Bronchiolar aerosol deposition and clearance}

\author{
J. E. Agnew
}

We have often, and over a long time, thought of the "small airways" as a mystery zone or a quiet zone [1]. Relative to central bronchial generations, the total crosssectional area of each bronchiolar generation is large. Airflow resistance should in consequence be low. Considerable changes due to disease could, thus, occur with little chance of being detected by conventional lung function testing. This is the basic idea of a "quiet zone" at risk of insidious damage. The concept was strengthened by the finding that early damage from cigarette smoking occurs within these airways [2]. However, even for the normal lung, there is morphological evidence that internal bronchiolar diameters appear to act as a major determinant of ventilatory function [3]. Conventional spirometry supposedly reflects "large airways" function, but is significantly related to specific pathological scores of small airways disease in patients with minimal airways obstruction [4]. Sustained exploration of "small airways tests" has suggested that several such tests can respond sensitively to bronchiolar inflammation [5]. Sadly, these tests generally lack predictive ability. In particular, they cannot foretell which smokers will develop overt disease [5].

Considerable attention has been paid to the small airways in asthma. Even when free from symptoms, asthmatics may well have persisting evidence of a small airways abnormality, at least partially reversible by bronchodilator administration [6]. A severe attack is likely to be accompanied by highly significant bronchiolar abnormalities including inflammation and lumen occlusion [7].

A different focus of attention on the small airways has recently come from the radiation safety field. Driven by concerns over risks from long-lived radioactive particulates retained in workers' lungs, a new respiratory tract model - from the International Commission on Radiological Protection (ICRP) - explicitly treats the bronchi and the bronchioles as separate zones [8]. These two zones are held to differ not only in their deposition and clearance characteristics but also in terms of the cellular sites considered to be crucially radiation-sensitive.

New methods of investigating the small airways are, therefore, to be welcomed. In this issue of the Journal, SVARTENGREN et al. [9] describe their evaluation of small airways deposition and clearance. The findings and approach of this study offer a chance to reconsider the "mystery zone" status of the small airways and to look again at their defence role in the lungs.

Correspondence: J.E. Agnew, Royal Free Hospital and School of Medicine, London NW3 2QG, UK.

\section{Deposition by very slow inhalation}

Recent aerosol work from the Karolinska Institute [10] has exploited a simple idea. The starting point is that, by means of a very slow inhalation, quite large aerosol particles (aerodynamic diameter $\sim 6 \mu \mathrm{m}$ ) can be persuaded to pass through the large airways with almost no deposition. This is achieved with airflow velocities just too low for particle deposition by inertial impaction. Within the small airways, due to their larger total cross-section, airflow velocities become lower still. Transit times through each individual airway unit are then long enough, and yet internal diameters small enough, for many particles to deposit by sedimentation under the influence of gravity. Calculations $[9,11]$ based on WeIBEL model geometry [12] demonstrate the magnitudes of the effects involved.

\section{Sites of long-term particle retention}

For many years a "Task Group" lung model, published in Health Physics in 1966 [13], defined lung zones for radioactive particle retention. Particles deposited within the lung could be attributed either to "tracheobronchial" or "alveolar" deposition. Calculations were kept simple by the concept that insoluble particles were cleared from the tracheobronchial airways by a fast clearance process - mucociliary transport. Clearance by this means was completed within $24 \mathrm{~h}$. By contrast, the alveolar zone was considered to be cleared only by slowly-acting mechanisms. Negligible alveolar clearance was, therefore, expected in the first $24 \mathrm{~h}$. A single measurement at $24 \mathrm{~h}$, as a percentage of initial lung deposition, hence served to measure "\% alveolar deposition". Insoluble particles still present at $24 \mathrm{~h}$ would be expected to be retained for a very long span of time. If they carried a long-lived radio-active content, they could well constitute a significant carcinogenic hazard.

The concept of the $24 \mathrm{~h}$ watershed between "tracheobronchial" and "alveolar" deposition encountered progressively strengthening criticism $[14,15]$. Even in healthy people, inhalation manoeuvres which encouraged deposition deep within the bronchial tree tended to give a degree of clearance from $24 \mathrm{~h}$ to 48 or $72 \mathrm{~h}$ too great to seem acceptable as early "alveolar" clearance rather than continuing "tracheobronchial" clearance. For patients with airways obstruction, retardation of tracheobronchial mucociliary transport must make any tracheobronchialalveolar clearance watershed even more difficult to pinpoint.

Twenty four hour incompleteness of tracheobronchial clearance is now, however, a minor part of the long-term 
retention story. Recently, major emphasis has been placed on retention at tracheobronchial sites $[8,16]$. The idea is not new but has gained impetus from inhalation studies using radioactive aerosol bolus techniques [17, 18], with support from studies aiming at bronchiolar deposition by the slow inhalation technique $[9,10]$. Utilizing either technique, the proposition is that the aerosol particles are carried only a fairly short distance into the bronchial tree and there undergo efficient deposition. The idea only makes good practical sense if the particles are large enough to undergo deposition fairly promptly. Particles not deposited via a prompt deposition mechanism could, of course, be carried much deeper into the lungs by gas-mixing mechanisms.

\section{Aerosol bolus and probe techniques}

When aerodynamic diameter (or actual diameter for material of "unit" density in grams per cubic centimetre) is about $0.5-1 \mu \mathrm{m}$, all the main particle deposition mechanisms are ineffectual. These particles are too small for appreciable inertial deposition, or gravitational sedimentation, unless a postinspiratory breathhold pause allows sufficient time for adequate sedimentation to occur. They are too large for appreciable deposition by diffusion. A bolus of such particles inhaled only to a shallow lung depth can (in the absence of a breathhold pause) readily be remeasured on exhalation but with its profile considerably broadened [19]. An inhaled bolus of width $50 \mathrm{~cm}^{3}$ (actually measured as "half-width" from a plot of aerosol concentration against inhaled/exhaled volume) inhaled to a volumetric depth within the lungs of $200 \mathrm{~cm}^{3}$ is "recovered" in the expired volume as a bolus of width $275 \mathrm{~cm}^{3}$ [19]. Mathematical modelling, based on the Weibel model, has up to a point agreed [20] with the experimental data, although with reservations over effects due to lung asymmetry.

When variable breathhold pauses are employed, efficiency of aerosol recovery following inhalation of larger aerosol volumes can be used to estimate airway diameters at different lung depths. Essentially, the argument is that the time required for a certain fraction of particles to be deposited is a function of the distance through which they have to fall. This, in turn, is a function of airway diameters at the lung volumetric depth in question. Experimental data depend, as expected, on the subject's degree of lung inflation and show good agreement with morphometric data [21]. Taken together, aerosol bolus and probe developments have added to the belief that we can now understand and predict particle aerodynamic behaviour within the lungs. They thus support the proposition that we can predictably target test particles onto the bronchiolar airways [9, 10].

\section{Ineffective bronchiolar mucociliary defence?}

Many particles deposited at bronchiolar sites seem to be retained for a long time $[9,10,17,18]$. Arguments for the seeming inefficiency of bronchial or of bronchiolar defence by mucociliary transport, thus, need to be reexplored. When the "mucociliary" clearance process takes 2 or 3 days $[9,10]$ to complete, we can reasonably accept that peripheral mucociliary rates are slower than had previously been thought. The greater worry, however, is the high proportion of particles appearing totally to escape mucociliary clearance.

Localized gaps in the mucous blanket can clearly constitute a "hole in the defence". They probably cause normal bronchioles to be poorly defended - at least against the retention of inert, nonirritant test particles. Early smoking-related bronchiolar changes [2] may, in fact, temporarily improve peripheral lung zone particle clearance, by making more mucus available for mucociliary transport [22].

Much more emphasis has recently been placed on particle transport into and through mucous layers [23]. Currently, this seems probably most relevant to longterm retention of small particles. The probability that an insoluble particle deposited in the bronchioles will be retained there for weeks seems to depend strongly on its geometric size [16, 18]. Surface tension forces appear far more likely to pull small rather than large particles through the mucous layers and into contact with the epithelial surface [23]. Retention within the normal lung $72 \mathrm{~h}$ postinhalation of about $60 \%$ has been reported for $2.4 \mu \mathrm{m}$ geometrical diameter particles deposited from a bolus inhaled only to a volumetric depth of about $60 \mathrm{~cm}^{3}$ [17]. Providing simple volumetric considerations apply, these particles should have been deposited primarily in bronchi and bronchioles. The very slow inhalation technique sets out to achieve predominantly bronchiolar deposition. Applied to $4.1 \mu \mathrm{m}$ geometrical diameter particles it yields a normal $72 \mathrm{~h}$ retention of about 36\% [10]. Setting aside different experimental conditions, these findings together imply that normal small airways have very imperfect mucociliary defence. The same finding appears also to apply in asthma [9] - again in terms of the proportion of particles not cleared by any reasonably fast clearance process. The only caveat has to be a lingering doubt that a considerable proportion of particles may, in fact, have actually reached the alveolated airspaces of the lung $[17,18]$. Marked dispersion of a nondepositing bolus [19] does suggest that particles at the front of the bolus may reach deeper into the lung than simple calculations imply, or that the lung's asynchrony may exert a considerable influence on where particles actually get to [19].

Interaction between lung structure and gravitational particle aerodynamics must also influence results obtained with the slow inhalation technique. Detailed studies of distal bronchiole deposition utilize quite sophisticated computer simulations. HofmAnN et al. [24] have estimated (by Monte Carlo methods) how large particles deposit under gravity in bifurcations at the terminal bronchiole level. Efficiency of deposition (summed over parent and daughter airways) was highest for a horizontally positioned bifurcation (parent and daughters all in the same horizontal plane). Efficiency could be as much as one-third lower for orientations in which the parent bronchiole was vertical [24]. Detailed deposition patterns were highly sensitive to bifurcation orientation [24]. These findings predict marked local variability in deposited surface concentrations. They also indicate a degree of localized variability in the proportion of particles able to escape deposition at this lung depth and, potentially, undergo deposition in the "alveolar region". 


\section{Deposition and clearance: asthmatics and normals}

A general view has been that mucociliary clearance tends to be impaired in asthma, albeit with a great variability among asthmatic subjects $[8,25]$. Even asthmatics apparently in clinical remission may show defective clearance [26]. For some asthmatics, the compensatory mechanism of cough can achieve reasonably normal aerosol clearance values - at least from the larger airways. For many asthmatics, bronchodilators may additionally stimulate clearance. Corticosteroid therapy can enhance peripheral zone radioaerosol clearance in asthma [27]. The results reported in this issue of the Journal [9] may, thus, demonstrate "normal" clearance running in parallel with good symptom control.

The new respiratory tract model [8] includes "modifying factors" both for disease and for medication effects. "Asthma"' is, within the model's framework, taken to slow bronchial and bronchiolar clearance by a factor of 0.7 . This factor is applied to the rate constant of an exponential equation. For bronchiolar clearance, a normal clearance half-time of about $8 \mathrm{~h}$ is, thereby, extended to an estimate for asthmatics of about $12 \mathrm{~h}$. In the formulation of the model, there is specific recognition that medication may enhance clearance rates [8] with, in fact, a proposed modifying factor for $\beta$-adrenergic agents of 2.0 (predominantly based on data from healthy subjects). What must be emphasized, however, is that the model's adoption of these factors is intended for radiation safety calculations covering populations likely to include asthmatic subjects. The model is based on "best currently available" estimates. It does not pretend to offer exact pathophysiological data. The results of SvARTENGREN $e t$ al. [9] for post-24 h clearance fit adequately comfortably with the generalised idea of the ICRP model - namely that clearance is achieved by a mixture of several "fast" and "slow" processes. They do, however, suggest that clearance between 48 and $72 \mathrm{~h}$ is similar to that between 24 and $48 \mathrm{~h}$. Mathematically, the model relying mainly (for normal subjects) on reference clearance rates corresponding to half-times of (roughly) $100 \mathrm{~min}, 8 \mathrm{~h}$ and $\geq 20$ h would predict rather less clearance over the 48 to 72 $\mathrm{h}$ interval. But again, it must be emphasized that the model only aims to give a general, and mathematically convenient, formulation of clearance dynamics.

\section{Bronchiolar or alveolar deposition?}

Disentangling bronchiolar from alveolar deposition is important when comparing measured retention data to calculated generation-by-generation deposition models [11]. These models have to make simplifying assumptions, not least in their utilization of simple deposition formulae for impaction and sedimentation. Designation of (say) generations 9-15 as "bronchiolar" [8] inevitably ignores the lungs' real asymmetry [28], let alone any question as to whether generation 15 should be considered "transitional" rather than "bronchiolar" [28]. Adjusting model dimensions to allow for differing degrees of lung inflation can also readily influence calculated deposition efficiencies.

Nevertheless, if we have to carry out safety calculations for a nuclear industry worker, we still want to know whether particles retained at 72 or $96 \mathrm{~h}$ are in the bronchioles or in the alveolar region. We need this knowledge as an input to basic radiation dose calculations and because it may help to predict whether retention will be long-term or very long-term. Likewise, when we consider possible targeted deposition of inhaled medication in asthma, we will want to know how much of an attempted bronchiolar deposition may actually overshoot into the alveolar region.

\section{Therapeutic implications?}

How helpful might it be to target therapeutic aerosol deposition selectively onto the small airways? If this is a desirable objective, can it be achieved in clinical practice by a very slow inhalation technique [9]? Methods of inhaled drug delivery are changing anyway [29], implying a need to look again at the better "targeting" of inhaled medication. The issues to be considered clearly include the target to be aimed at and the tightness of aim needed. It should, probably, be equally important to consider whether we have any means of verifying that the inhaled medication really is deposited on target.

Practical limitations have to be taken into account. Simple observation shows that inhaled drug delivery in a nonspecialist hospital ward tends to be a "hit-or-miss" affair. Careful audit has documented wide variability of practice, even after positive steps to advocate adherence to guidelines [30]. Despite careful attention to technique, supposedly similar devices may, in fact, deliver different drug amounts and with a varying spectrum of particle sizes.

For inhaled corticosteroids, as for bronchodilators, a guiding principle has been that an "ideal inhaler" should maximize conducting airways deposition, whilst minimizing both oropharyngeal and alveolar deposition [29]. For most drugs, there is, as yet, little knowledge - and certainly no consensus - as to whether peripheral rather than central airways deposition could really confer any benefit [29]. Aerosol deposition modelling, moreover, highlights how a shift from central to peripheral airways deposition can enormously change surface concentration of the deposited drug [11]. Many drug-receptor interactions are concentration-dependent, and a recent review in the Journal clarifies how such dependence may be implicated in hitherto inadequately understood consequences of long-term $\beta_{2}$-agonist use [31]. Attempts to target inhalation therapy onto the small airways should, therefore, be appropriately backed-up by long-term efficacy assessments.

\section{The small airways}

Various applications of sophisticated technology are currently carrying forward our understanding of the lung's quiet zone. Acoustic reflection work has suggested that a greater bronchodilator efficacy in asthma of adrenergic as opposed to anticholinergic bronchodilators may result from a greater effect specifically within the small airways [32]. Tomographic gamma-camera imaging aligned with data from magnetic resonance imaging has been used to map out particle deposition per airway generation 
in the clear hope of contributing to future targeted delivery of inhaled medication [33]. Differing aerosol tracer approaches have looked at deposition, clearance rates and long-term retention in the small airways $[9,10,17]$. Ideas from a respiratory tract model basically formulated for radiation safety needs [8] clearly interact with all the considerations involved. This model has purposely been so designed that it can take account of new data - most easily in the form of new or revised "modifying factors". Future work with this model will benefit from testing model predictions against real data and, when appropriate, modifying the model. Problems will remain over elucidating whether particles, inhaled according to one protocol or another, really are deposited in ciliated small airways or whether they may actually "overshoot" and, therefore, reach the alveolar zone. Further consideration of the lung's asymmetry should be helpful. Modelling based on symmetrical geometry tends to underestimate inhaled bolus dispersion [20], and may similarly tend to underestimate the proportion of particles capable of reaching alveolar generations via a "short" pathway or because the lung behaves asynchronously. Surface concentrations of deposited drug may be important determinants of effectiveness, or even of unwanted interactions [31]. Even using a symmetrical model, a shift in deposition from large to small airways sites is likely considerably to lessen particle surface concentrations [11]. When asymmetry is taken into account, mathematical modelling can further show that the degree of asymmetry profoundly affects localized deposition concentrations immediately distal to an airways bifurcation [34].

Large, or fairly large, inhaled particles have high deposition probabilities in the terminal bronchioles and adjacent airways. The slow inhalation technique $[9,10]$ encourages their deposition at the distal margins of the lung's "quiet zone" and, linked to findings from other approaches, must surely encourage our better understanding of that zone's function.

\section{References}

1. $\quad$ Mead J. The lung's "quiet zone". N Engl J Med 1970; 282: 1318-1319.

2. Niewoehner DE, Kleinerman J, Rice DB. Pathologic changes in the peripheral airways of young cigarette smokers. N Engl J Med 1974; 291: 755-758.

3. Niewoehner DE, Knoke JD, Kleinerman J. Peripheral airways as a determinant of ventilatory function in the human lung. J Clin Invest 1977; 60: 139-151.

4. Matsuba K, Shirakusa T, Kuwano K, Hayashi S, Shigematsu N. Small airways disease in patients without chronic airflow limitation. Am Rev Respir Dis 1987; 136: 1106-1111.

5. Buist AS. Current status of small airways disease. Chest 1984; 86: 100-105.

6. McCarthy D, Milic-Emili J. Closing volume in asymptomatic asthma. Am Rev Respir Dis 1973; 107: 559-570.

7. Saetta M, Di Stefano A, Rosina C, Thiene G, Fabbri LM. Quantitative structural analysis of peripheral airways and arteries in sudden fatal asthma. Am Rev Respir Dis 1991; 143: 138-143.

8. International Commission on Radiological Protection (ICRP). Human respiratory model for radiological protection (ICRP Publication 66). Ann ICRP 1994; 24: 1120.
9. Svartengren K, Philipson K, Svartengren M, Anderson M, Camner P. Tracheobronchial deposition and clearance in smaller airways in asthmatic subjects. Eur Respir J 1996; 9: 1123-1129.

10. Anderson M, Philipson K, Svartengren M, Camner P. Human deposition and clearance of $6 \mu \mathrm{m}$ particles inhaled with an extremely low flow rate. Exp Lung Res 1995; 21: 187-195.

11. Gerrity TR, Lee PS, Hass FJ, Marinelli A, Wemer P, Lourenco RV. Calculated deposition of inhaled particles in the airway generations of normal subjects. $J$ Appl Physiol: Respirat Environ Exercise Physiol 1979; 47: 867-873.

12. Weibel ER. Morphometry of the Human Lung. Berlin, Springer-Verlag, 1963.

13. Task Group on Lung Dynamics. Deposition and retention models for internal dosimetry of the human respiratory tract. Health Phys 1966; 12: 173-207.

14. Smaldone GC, Perry RJ, Bennett WD, Messina MS, Zwang J, Ilowite J. Interpretation of "24 hour lung retention" in studies of mucociliary clearance. J Aerosol Med 1988; 11-20.

15. Stahlhofen W, Rudolf G, James AC. Intercomparison of experimental regional aerosol deposition data. J Aerosol Med 1989; 2: 285-308.

16. Bailey MR, Dorrian M-D, Birchall A. Implications of airway retention for radiation doses from inhaled radionuclides. J Aerosol Med 1995; 8: 373-390.

17. Scheuch G, Philipson K, Falk R, et al. Retention of particles inhaled in boli with and without induced bronchoconstriction. Exp Lung Res 1995; 21: 901-916.

18. Stahlhofen W. Perspectives and considerations. J Aerosol Med 1995; 8: 402-404.

19. Anderson PJ, Hardy KG, Gann LP, Cole R, Hiller FC. Detection of small airway dysfunction in asymptomatic smokers using aerosol bolus behavior. Am J Respir Crit Care Med 1994; 150: 995-1001.

20. Darquenne C, Paiva M. One-dimensional simulation of aerosol transport and deposition in the human lung. $J$ Appl Physiol 1994; 77: 2889-2898.

21. Brand P, Rieger C, Beinert T, Heyder J. Aerosol-derived airway morphometry in healthy subjects. Eur Respir $J$ 1995; 8: 1639-1646.

22. Agnew JE, Lopez-Vidriero MT, Pavia D, Clarke SW. Functional small airways defence in symptomless cigarette smokers. Thorax 1986; 41: 524-530.

23. Gehr P, Schurch S, Berthiaume Y, Im Hof V, Geiser M. Particle retention in airways by surfactant. J Aerosol Med 1990; 3: 27-43.

24. Hofmann W, Balásházy I, Koblinger L. The effect of gravity on particle deposition patterns in bronchial airway bifurcations. J Aerosol Sci 1995; 26: 1161-1168

25. O'Riordan TG, Zwang J, Smaldone GC. Mucociliary clearance in adult asthma. Am Rev Respir Dis 1992; 146: 598-603.

26. Pavia D, Bateman JRM, Sheahan NF, Agnew JE, Clarke SW. Tracheobronchial mucociliary clearance in asthma: impairment during remission. Thorax 1985; 40: 171175.

27. Agnew JE, Bateman JRM, Pavia D, Clarke SW. Peripheral airways mucus clearance in stable asthma is improved by oral corticosteroid therapy. Bull Eur Physiopathol Respir 1984; 20: 295-301.

28. Weibel ER. Design of airways and blood vessels as branching trees. In: Crystal RG, West JB, eds. The Lung: Scientific Foundations. New York, Raven Press, 1991; pp. 711-720. 
29. Derom E, Pauwels R. Bioequivalence of inhaled drugs. Eur Respir J 1995; 8: 1634-1636.

30. Caldwell NA, Milroy R. Optimizing nebulization practice within a large teaching hospital: easier said than done. Respir Med 1995; 89: 57-59.

31. Adcock IM, Stevens DA, Barnes PJ. Interactions of glucocorticosteroids and $\beta_{2}$-agonists. Eur Respir J 1996; 9: $160-168$

32. Hoffstein V, Zamel N, McClean P, Chapman KR. Changes in pulmonary function and cross-sectional area of trachea and bronchi in asthmatics following inhalation of procaterol hydrochloride and iptratropium bromide. $A m J$ Respir Crit Care Med 1994; 149: 81-85.

33. Fleming JS, Nassim M, Hashish AH, et al. Description of pulmonary deposition of radiolabeled aerosol by airway generation using a conceptual three dimensional model of lung morphology. J Aerosol Med 1995; 8: 341'-356.

34. Balashazy I, Hofmann W. Deposition of aerosols in asymmetric airway bifurcations. J Aerosol Sci 1995; 26: 273-292. 